\title{
The Metabolic Syndrome Real or Myth
}

\author{
Reginald Walter Marsh* \\ Professor, Waikato Clinical School, Auckland University, New Zealand
}

*Corresponding author: Reginald Walter Marsh, Professor, Waikato Clinical School, Auckland University, 480c Devonport Road, Tauranga South 3112, New Zealand, Tel: +64 7 5716735; E-mail: marshrw@hotmail.com

Received: 02 Jan, 2021 | Accepted: 12 Jan, 2021 | Published: 16 Jan, 2021

Citation: Marsh RW (2021) The Metabolic Syndrome Real or Myth. J Heart Health 7(1): dx.doi.org/10.16966/2379-769X.160

Copyright: (c) 2021 Marsh RW. This is an open-access article distributed under the terms of the Creative Commons Attribution License, which permits unrestricted use, distribution, and reproduction in any medium, provided the original author and source are credited.

Assumptions underlying the concept of the Metabolic Syndrome (MS) are implicit rather than explicit. Categorization of patients into have and don't have MS groups is based on five features, any combination of three or more may be used to determine which category they belong to. These features are triglyceride, glucose, and High Density (HD) cholesterol levels, blood pressure and obesity.

The number of combinations arising from five features taken three at a time is found by substituting in the following expression:

$\mathrm{n} ! / \mathrm{k} !(\mathrm{n}-\mathrm{k})$ !; thus 5 ! /3!(5-3)!=120/12=10

These 10 combinations can be characterized as: $\mathrm{ABC}, \mathrm{BCD}, \mathrm{BDE}$, $\mathrm{CDE}, \mathrm{BAD}, \mathrm{CAD}, \mathrm{CAE}, \mathrm{DEA}, \mathrm{ABE}, \mathrm{BCE}$ where Obesity=A; $\mathrm{BP}=\mathrm{B}$; HD Cholesterol $=\mathrm{C}$; Triglycerides $=\mathrm{D} \&$ Glucose $=\mathrm{E}$.

If the metabolic syndrome is a coherent entity the assumption is that any of these combinations of variables will produce very similar categorizations regardless of which of the 10 combinations is used. This is largely true but the above analysis also shows that while the categorizations correctly identify about $58 \%$ of the actual cases they also incorrectly identify many others (43\%) (See table 1 below). I used data from the well known U.K. Caerphilly study. This does not include glucose levels so I could use up to 4 features only. But it has the advantage of noting an outcome of Myocardial Infarct (MI) followed for over 30 years, which allows verification of the results. The discriminant function technique (SPSS v.26) was used.

False positives are those cases misclassified as associated with MI when they are not and false negatives are the misclassification as not associated with MI when, in fact, they are.
It is clear from the results that the classification, using these defining variables, of having the metabolic syndrome is less than $10 \%$ better than chance $(50 / 50)$. The number of false positive selections is about three times as great as the true positives which account for only $58 \%$ of the actual positive cases. The amount of total variance accounted for by these defining variables ranges between $23 \%$ to $34 \%$. When four definers $\mathrm{ABCD}$ were used together the results were only marginally better.

This weak relationship between MI and defining variables demonstrates that the metabolic syndrome is a very imperfect concept. In its present form its use is likely to give misleading conclusions.

Thanks to Professor Y. Ben-Shlomo for access to the Caerphilly data.

Table 1: Results of applying three groups of classifiers to data on myocardial infarcts (Of the actual 1786 cases; 348 had MI and 1438 did not have $\mathrm{MI}$ ).

\begin{tabular}{|l|c|c|c|c|c|}
\hline \multicolumn{7}{|c|}{ Groups } \\
\hline \multicolumn{1}{|c|}{ Classifiers } & ABC & BCD & BAD & CAD & ABCD \\
\hline True Positives & $201(58 \%)$ & 199 & 199 & 198 & 203 \\
\hline False Positives & 622 & 585 & 570 & 607 & 586 \\
\hline True Negatives & $816(57 \%)$ & 853 & 868 & 831 & 852 \\
\hline False Negatives & 147 & 149 & 149 & 150 & 145 \\
\hline
\end{tabular}

